\title{
A public-private partnership model for COVID-19 diagnostics
}

\section{To the Editor - The SARS-CoV-2}

pandemic placed unprecedented pressure on diagnostic systems worldwide. In early 2020 , most countries were completely unprepared to carry out the massive systematic testing of symptomatic and asymptomatic individuals necessary to monitor and control spread of a virus undergoing community transmission. Not only were the established procedures for viral molecular diagnostics, which are relatively low throughput and labor intensive, not designed for testing at this scale, but also the testing procedures in clinical laboratories often relied on reagents from a single supplier. Together, these factors quickly resulted in inadequate testing capacity, shortages of testing consumables, unsupportable workloads for diagnostic personnel and a breakdown in diagnostic healthcare services.

To meet this challenge, many academics and research institutes around the world set up their own ad hoc testing facilities (for example, the Broad Institute's (https://www. broadinstitute.org/coronavirus/covid-19) and refs. ${ }^{1,2}$ ), providing urgently needed testing capacity to local communities. In addition, government initiatives and funding were rapidly established to galvanize the creation of innovative diagnostic assays capable of meeting the staggering demand of a novel pathogen undergoing community transmission (for example, RADx (https://www.nih.gov/research-training/ medical-research-initiatives/radx) in the United States). Here, we report on another approach: the creation of an ad hoc public-private consortium that was able to rapidly design, develop and implement a high-throughput diagnostic platform for SARS-CoV-2, enabling testing on a massive scale in the Netherlands. This consortium may provide a model for other countries seeking to rapidly build capacity in diagnostic testing for COVID-19 and for other infectious diseases.

In March 2020, some of us (P.H.L.K., T.A.H., S.B., M.P., P.W.T., I.L., B.M.P.V., M.J.A.M.V., T.W.v.R., F.M., W.d.L. and M.E.T.) sought to exploit our molecular biology expertise at the Hubrecht Institute, an academic institute, to contribute to efforts to increase Dutch testing capacity for SARS-CoV-2. Realizing that rapid development of major diagnostic innovations would require cross-disciplinary expertise, we reached out to experts in laboratory automation at the Utrecht-based biotech company Genmab (R.R. and M.B.). Together, we concluded that modifications to the entire testing procedure, including sample collection, were required to realize a transformative increase in diagnostic throughput.

Initially, Dutch clinical diagnostics experts and policymakers regarded our proposal to completely overhaul the entire SARS-CoV-2 diagnostic testing procedure into a highly automated, low-cost PCR testing platform as too risky and outside-the-box; instead, they elected to immediately scale up existing infrastructure-a less risky, tried-and-tested approach-as a means of rapidly increasing testing capacity. Nevertheless, we remained convinced of the value of our high-throughput platform, and the public and private partners in our consortium (Hubrecht Institute and Genmab, respectively) continued our efforts to build it out using financial support from several non-profit institutions, including the Oncode Institute, a Dutch cancer research institute wishing to relieve the COVID-19imposed burden on cancer healthcare services, as well as from the Royal Dutch Academy of Sciences (KNAW) and the Friends of the Hubrecht Foundation. For further development and implementation of the newly designed high-throughput testing platform, we established new contacts with experts at clinical diagnostics centers (L.I.P.M.D., M.M.C.B., R.J.T.M.R., L.B.J.v.d.V. and J.H.B.v.d.B. at the Laboratory for Pathology and Medical Microbiology (PAMM) and A.v.W. at Sanquin Diagnostics) and a clinical information technology specialist company (E.D. at Bodegro). Having been involved in COVID-19 testing during the first wave of infections, these diagnostic partners recognized the importance of innovation for scaling up COVID-19 diagnostics. Both academic and industry partners shared a spirit of open innovation, a very positive mindset, and a hands-on style of working, and the new consortium members supported the clinical implementation of the new testing platform. Municipal Health Services (G.G.D.), which is responsible for COVID-19 sample collection in the Netherlands, was recruited by PAMM soon after, as was Tecan (S.M.), which is a manufacturer of custom robotic devices.
Under normal circumstances, collaborations between academia, biotech, diagnostics and healthcare workers are rare and often difficult to establish rapidly due to complex collaboration agreements, but uniting these traditionally poorly connected disciplines was a necessity if we were to succeed in rapidly developing and implementing our platform. Given the urgency of the situation, formation of this consortium was based solely on trust and a strong mutual desire to contribute to society without personal or institutional gains, a goal that was broadly supported by the leadership of all the organizations involved-whether public or private. As such, the consortium acted in lieu of written agreements, which allowed very rapid establishment of the consortium and its activities. Ultimately, our ad hoc consortium was able to rapidly put in place our optimized testing platform, Systematic Testing using Robotics and Innovation during Pandemics (STRIP): it took 2-3 months for assay development, 6 months for delivery of the liquid handler (an extended duration due to global shortages in equipment) and a further 2 months for optimization and clinical validation.

STRIP is a start-to-end streamlined and automated procedure for COVID-19 testing, centering on a single Tecan Fluent liquid-handling robot that can process $>14,000$ samples per day (Fig. 1). The sensitivity, specificity and practical implementation of STRIP have been validated in a clinical study on 1,128 individuals, meeting the standards set by the Dutch National Institute for Public Health and the Environment (playing a similar role to the United States' Centers for Disease Control and Prevention; https://lci.rivm.nl/ covid-19/bijlage/aanvullend). Automation throughout the testing procedure dramatically reduces the workload on diagnostic laboratory personnel and potentially allows the placement of multiple STRIP liquid-handling robots per testing facility, further increasing testing capacity. The entire test procedure also requires only three pipet tips per sample, as well as reduced testing reagents via process miniaturization, which is important given scarcity of testing consumables during the COVID-19 pandemic. Furthermore, STRIP is compatible with reagents from any 

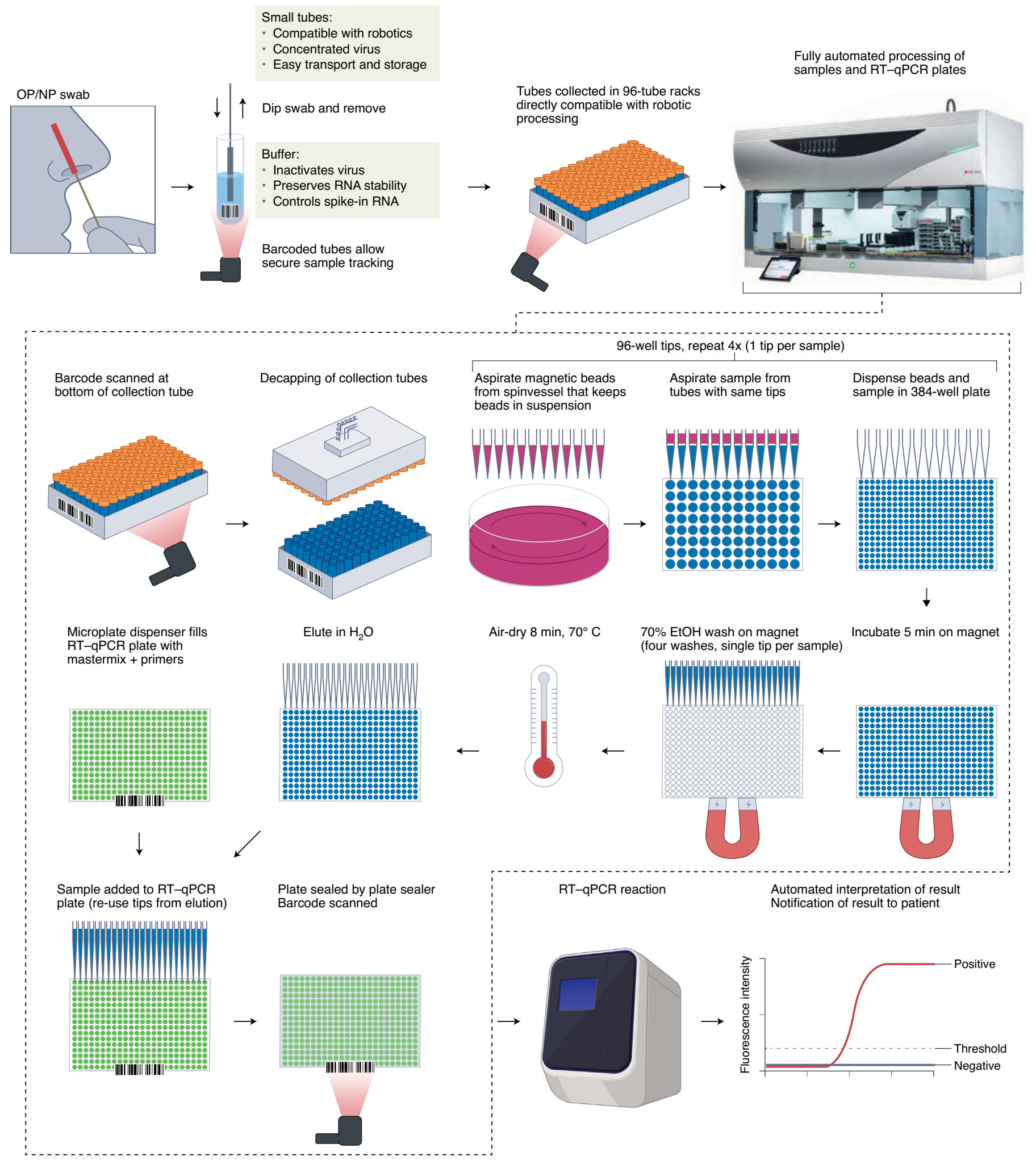

Fig. 1 | Schematic overview of the STRIP workflow. OP, oropharyngeal; NP, nasopharyngeal; RT-qPCR, reverse transcription quantitative PCR.

supplier and thus less sensitive to supply chain bottlenecks. Finally, the system is open and modular, facilitating adaptation of future developments in diagnostics. Overall, the system enables substantial savings in personnel and reagents requirements compared with conventional diagnostic testing; when STRIP runs at full capacity, it is possible to rapidly (within days) recoup the initial outlay in the liquid-handling system from savings in personnel costs, reagents and materials. 
To achieve the very high-throughput sample processing of the STRIP procedure, we implemented several engineering innovations (Fig. 1). These innovations required modifications to the molecular biology of the test, thus requiring close collaboration among automation experts and molecular biologists. Similarly, automated sample tracking required collaborations among automation experts and information technology specialists, and practical implementation of the entire procedure required collaboration among clinical diagnostics experts and assay developers. For example, one of the most critical innovations within STRIP is the collection of samples in small tubes $(0.8$ $\mathrm{ml}$, as compared with 5-15 $\mathrm{ml}$ in standard diagnostics) with virus-inactivating and RNA-stabilizing lysis buffer (provided by InActiv Blue). The use of small tubes enables collection of sample tubes in small 96-tube boxes at sample collection sites. These 96-tube boxes are directly compatible with automated sample processing in the lab without slow and often laborious transfer of samples from collection tubes to plates that are compatible with robotics. Integration of small tubes into the testing procedure required adjustments to the molecular biology, automation and diagnostic laboratory procedures, as well as to sample collection protocols performed by public health services, highlighting the importance of a multidisciplinary consortium for rapid implementation of diagnostic innovations.

During the second wave of infections, in the fall of 2020, the Dutch government adopted development of STRIP, and the platform is now operating within the Dutch national COVID-19 testing infrastructure coordinated by the Dutch Ministry of Health. At least five more STRIP robots have been ordered by the Dutch government. These will form a nationwide infrastructure, part of a preparedness plan for future pandemics. Design, protocols and automation scripts are open source and are available through Protocols.io at https://doi. org/10.17504/protocols.io.bxiwpkfe.

We believe that the STRIP platform is a viable means of supplementing existing testing infrastructure. It can help rapidly scale COVID-19 testing and can be of importance to societies worldwide for the management of future pandemics. Moreover, we hope that the STRIP consortium-a non-profit public-private partnership based on mutual trust and a shared desire to help society-can also provide inspiration for formation of similar collaborations worldwide when urgent societal needs must be addressed that cannot be tackled adequately by any single organization.

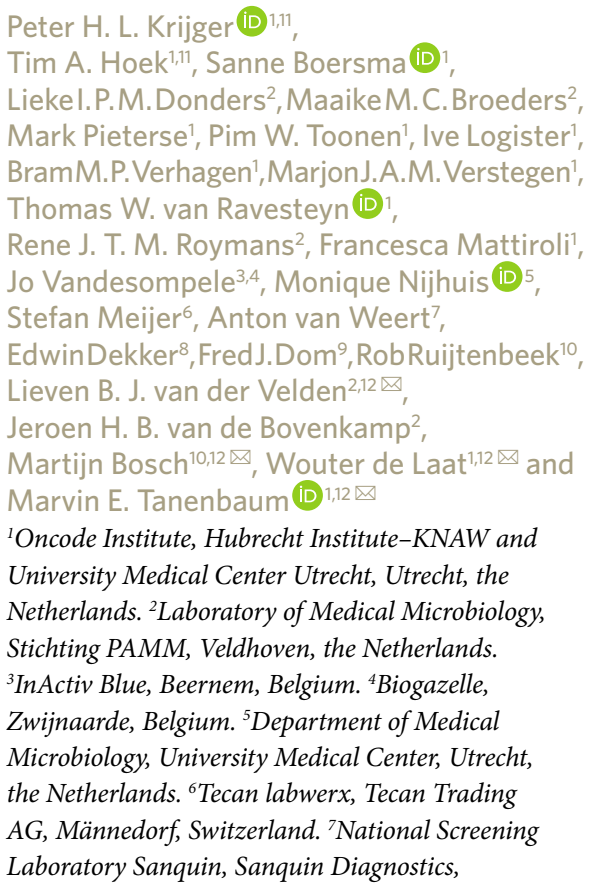

Amsterdam, the Netherlands. ${ }^{8}$ Bodegro B.V, Breda, the Netherlands. ${ }^{9} \mathrm{HiFiBio}$ Therapeutics, Saint-Jacques, France. ${ }^{10}$ Department of Laboratory Automation, Genmab B.V., Utrecht, the Netherlands. ${ }^{11}$ These authors contributed equally: Peter H. L. Krijger, Tim A. Hoek. ${ }^{12}$ These authors jointly directed this work: Lieven B. J. van der Velden, Martijn Bosch, Wouter de Laat, Marvin E. Tanenbaum.

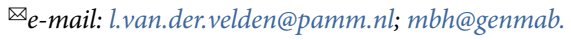
com; w.laat@hubrecht.eu; m.tanenbaum@hubrecht.eu

Published online: 28 September 2021 https://doi.org/10.1038/s41587-021-01080-6

References

1. Aitken, J. et al. Nat. Biotechnol. 38, 927-931 (2020).

2. IGI Testing Consortium. Nat. Biotechnol. 38, 791-797 (2020).

\section{Acknowledgements}

We thank the Foundation Friends of the Hubrecht Institute, the Royal Dutch Academy of Sciences (KNAW), Health $\sim$ Holland Top Sector Life Sciences \& Health, and the Oncode Institute for financial support. We thank Erik van de Linde, members of the VWS Taskforce STRIP- 1 and Niek Dubelaar for support. We thank all the members of the Hubrecht Institute who were involved in the early stages of the project, Peter Bredenbeek for SARS-CoV-2 control plasmids and Sietske Bakker for facilitating contact between the Hubrecht Institute and Genmab. We thank G.G.D. Brabant-Zuidoost for help with the collection of clinical specimens for COVID-19 testing. M.N. acknowledges support from Health Holland Clear Covid-19 (LSHM20058) and ZonMw project 114025009. We also thank members of the UMC Utrecht, especially Aniek Janssen, Maria Rodriguez-Colman, Jurian Schuijers, Andreas Sonnen and Rob Schuurman, for discussions. Finally, we thank the Dutch public test service (dienst testen) for support.

\section{Author contributions}

P.H.L.K., T.A.H, W.L. and M.E.T. wrote the manuscript with input from all other authors.

\section{Competing interests}

J.V. is co-founder and CSO of InActiv Blue; S.M. is an employee of Tecan Trading AG; L.I.M.P.D., M.M.C.B., R.J.T.M.R., L.B.J.v.d.V. and J.H.B.v.d.B. are employees of Stichting PAMM Veldhoven; A.v.W. is an employee of Sanquin; E.D. is a founder and employee of Bodegro; and F.J.D. is $\mathrm{CEO}$ and co-founder HiFIBiO France. 